\title{
DEL CIELO Y LA ATMÓSFERA DE CANARIAS: LA CREACIÓN DEL INSTITUTO DE ASTROFÍSICA DE CANARIAS
}

\author{
Fernando de Ory Ajamil* \\ Universidad de La Laguna
}

\section{RESUMEN}

Desde el comienzo del siglo Xvir son conocidas las excepcionales condiciones del cielo y la atmósfera en las cumbres del archipiélago canario. En un tiempo muy posterior, a mediados del siglo xx, el actual Instituto de Astrofísica de Canarias (IAC) surgió en el Observatorio Meteorológico de Izaña (OMI). En sus dependencias se alojaron y trabajaron los primeros investigadores del IAC. El primer edificio del Observatorio Astronómico del Teide (OAT) y el suministro eléctrico para su funcionamiento fueron sufragados por el OMI. Gran parte del personal del OMI trabajó y colaboró en las primeras observaciones del OAT. La larga serie de observaciones climatológicas del OMI constituyó la base y el fundamento sobre el que se justificó la idoneidad del emplazamiento para la investigación astronómica. Así sucedió en los primeros años, posteriores a 1959, cuando se creó oficialmente el OAT. Palabras clave: Izaña, Teide, observatorio meteorológico, astrofísico.

\author{
FROM THE SKY AND ATMOSPHERE OF THE CANARIAS: \\ THE CREATION OF THE INSTITUTO DE ASTROFÍSICA DE CANARIAS
}

\section{Abstract}

Since the beginning of the seventeenth century the exceptional conditions of the sky and the atmosphere on the summits of the Canary Islands archipelago have been known. Much later time, in the middle of the 20th century, the current Instituto de Astrofísica de Canarias (IAC) emerged at the Meteorological Observatory of Izaña (OMI). The first researchers of the IAC stayed and worked in its installations. The first building of the Observatorio Astronómico del Teide (OAT) and the electricity supply for its operation were financed by OMI. Much of the OMI staff worked and collaborated on the first observations of the OAT. The long series of IMO climate observations provided the basis and basis on which the suitability of the site for astronomical research was justified. This was the case in the early years, after 1959, when the OAT was officially established.

Keywords: Izaña, Teide, Observatory Meteorological, Astrophysic. 


\section{INTRODUCCIÓN}

Las islas Canarias reúnen en su fragmentado y reducido territorio una serie de excepcionales condiciones donde se conjuga la singularidad con la multiplicidad de oportunidades para el estudio y el ensayo de diversas experiencias en todas y cada una de las disciplinas científicas relativas a la naturaleza: meteorología, astrofísica, botánica, entomología, vulcanología, oceanografía, etc.

Así ha sido reconocido prácticamente durante los últimos cuatro siglos, cuando estas disciplinas científicas surgieron como tales y se configuraron a partir de numerosas e importantes experiencias llevadas a cabo en la tierra, el mar y el aire del archipiélago canario ${ }^{1}$.

Digamos a modo de recapitulación que el Observatorio Meteorológico de Izańa, el primero de la actual AEMET, fue inaugurado el 1 de enero de 1916, mientras que el Observatorio Astronómico del Teide, germen del IAC, fue creado por orden del ministro de Educación publicada en el BOE de 10 de febrero de 1959. Pero no fue hasta el 16 de septiembre de 1975 cuando se crearía el Instituto de Astrofísica de Canarias mediante un acuerdo con el Consejo Superior de Investigaciones Científicas (CSIC) y la Universidad de La Laguna (ULL), además de los cabildos insulares de La Palma y Tenerife.

\section{CIENCIA Y SOCIEDAD EN ESPAÑA ENTRE 1940 Y 1960}

Las condiciones de vida en España tras la guerra civil y la posguerra quedaron tan devastadas a su paso que los indicadores socioeconómicos previos a la contienda no se recuperaron hasta finales de 1950. Las consecuencias, que afectaron en todos los órdenes de la sociedad española, se dejaron sentir igualmente en el «microcosmos de Izaña». Aunque paradójicamente fue entonces, en la década de los años cincuenta, cuando sucedió la creación del Observatorio Astronómico del Teide y otros análogos.

Se trata de dos décadas en las que las cartillas de racionamiento eran moneda corriente -la Comisaría de Abastos de Tenerife provisionaba mensualmente al personal de OAI una precaria lista de alimentos básicos-, la carencia de combustible imponía restricciones a la protección del frío y al transporte de personas y bienes -como se comprobará en el suministro eléctrico al OAT-, el pluriempleo en la

* Doctor en Historia por la ULL, profesor de la EUPAM (ULL) y observador meteorológico de AEMET.

${ }^{1}$ En diversas publicaciones he enumerado muchos de estos estudios y experiencias llevadas a cabo por innumerables naturalistas y científicos europeos. Véanse en De OrY AJAmiL $(1997,2005)$ y en los «Calendarios Meteorológicos de AEMET» de los años 1994, 1995, 2005. En Ciencia y diplomacia hispano alemana en Canarias: el origen del observatorio meteorológico de Izaña (1909-1916), Ed. Edirca, Las Palmas de Gran Canaria (1997) o en Ciencia y presencia extranjera en Canarias. Desde la Ilustración a la Primera Guerra Mundial, Ed. Idea, Santa Cruz de Tenerife (2004). 
administración era el modo de supervivencia de las magras clases medias -característica igualmente del personal de ambos observatorios-y el ambiente social y laboral estaba teńido de un ordenancismo militar que se palpaba en todos los ámbitos -como es visible en multitud de documentos del OAI, pues no en vano formó parte como servicio militarizado del Ejército del Aire desde 1940 hasta 1978-.

En el orden científico la política del Estado franquista extendió muy rápida e inexorablemente las depuraciones y purgas que practicó en toda la administración pública. Como consecuencia de ello, de 580 catedráticos que había en las doce universidades españolas, 20 fueron asesinados, 150 expulsados y 195 se exiliaron ${ }^{2}$. Además de estos cargos universitarios, hubo otros muchos científicos españoles de primera fila -Giral los cifra en más de 500- que se exiliaron al acabar la guerra ${ }^{3}$. En la nómina de astrónomos espańoles exiliados, y otros tantos meteorólogos, cabe enumerar a Honorato Castro Bonell, Pedro Carrasco Garrorena, Amós Sabrás Gurrea y Marcelo Santaló Haro.

El que fuera ministro de Educación de Franco entre 1939 y 1951 asumió la decisión de «recristianizar la sociedad». Bajo la férrea mano del sacerdote y miembro del Opus Dei José María Albareda se constituyó en 1939 el Consejo Superior de Investigaciones Científicas, que depuso a la Junta de Ampliación de Estudios, de raíz republicana. Según la orden de 24 de noviembre de aquel año, el CSIC se creó para "cimentar ante todo la restauración de la clásica y cristiana unidad de las ciencias destruida en el siglo XVIII». Para ello había que

subsanar el divorcio y discordia entre las ciencias especulativas y experimentales y promover en el árbol total de la ciencia su armonioso incremento y su evolución homogénea, evitando el monstruoso desarrollo de algunas de sus ramas, con anquilosamiento de otras. Hay que crear un contrapeso frente al especialismo exagerado y solitario de nuestra época, devolviendo a las ciencias su régimen de sociabilidad, el cual supone un franco y seguro retorno a los imperativos de coordinación y jerarquía. Hay que imponer, en suma, al orden de la cultura, las ideas esenciales que han inspirado nuestro Glorioso Movimiento, en las que se conjugan las lecciones más puras de la tradición universal y católica con las exigencias de la modernidad ${ }^{4}$.

En suma, como analiza y demuestra un trabajo del profesor Otero Carvajal ${ }^{5}$, la guerra civil frenó en seco la consolidación de un sistema científico en España, cuyas bases se habían sentado a lo largo del primer tercio del siglo xx gracias a la labor de la Junta de Ampliación de Estudios. Las bases ideológicas y culturales de la dictadura franquista representaron un retroceso de alcance histórico para el débil y frágil entramado científico español. El exilio significó la sangría de una parte sus-

2 Castillo Martos, M. y Rubio Mayoral, J.L. (2014). Enseñanza, ciencia e ideología en España (1890-1950), ed. Diputación de Sevilla y Vitela Gestión Cultural. Sevilla.

${ }_{3}$ Giral, F. (1994). Ciencia española en el exilio (1939-1989), ed. Antrophos.

${ }^{4}$ BOE. 28.11.1939.

5 Otero Carvajal, L. (2006). La destrucción de la ciencia en España: depuración universitaria en el franquismo. Ed. Universidad Complutense. Madrid. 
tancial del capital humano de la cultura española, incluido el componente científico, dando lugar a una descapitalización que tardó decenios en ser recompuesta. Las depuraciones de maestros, profesores de bachillerato, profesores universitarios y científicos excluyeron de la práctica profesional a miles de personas capacitadas, cuyo coste ha sido insuficientemente ponderado hasta el momento para el desarrollo educativo, la formación y la calificación de la sociedad espańola de la larga posguerra.

\section{LOS INSPIRADORES DEL OBSERVATORIO ASTRONÓMICO DEL TEIDE. PRIMEROS PASOS}

El sacerdote jesuita Antonio Romañá Pujó (Barcelona 1900-1981), doctor en Ciencias Exactas y director del Observatorio de Física Cósmica del Ebro, siguiendo la propuesta de José María Albareda, creó en 1946 dentro del CSIC la Unión Nacional de Astronomía y Ciencias Afines (UNACA). Teniendo en cuenta que desde 1944 las enseñanzas de Astronomía General y Topografía, Astronomía Esférica y Geodesia se reducían a unas pocas cátedras en Madrid, Barcelona, Zaragoza, Santiago de Compostela y Oviedo, la idea de este centro es que fuera una especie de departamento de Astronomía dentro del $\mathrm{CSIC}^{6}$.

Para ello contó con la estrecha colaboración de José María Torroja Menéndez. Natural de Madrid (1916-1994), Torroja leyó su tesis doctoral en ciencias exactas con 25 ańos en el Observatorio del Ebro, bajo la dirección del jesuita Enrique de Rafael. En este observatorio conoció a Antonio Romañá. Respondiendo al perfil de la época, Torroja se empleó en todas las esferas a su alcance: catedrático de universidad, astrónomo del Observatorio Astronómico de Madrid, ingeniero geógrafo del IGN, consejero del CSIC, decano y vicerrector de la UCM, miembro y luego secretario de la Real Academia de Ciencias Exactas, Físicas y Naturales, y un largo etcétera. Como si encarnara el espíritu del decreto de creación del CSIC, la diversidad de tareas a las que se dedicó le proporcionó un conocimiento de carácter enciclopédico que le impidió sobresalir como especialista en un campo concreto.

Por ser ajena a su formación de matemático, la astrofísica no fue la disciplina que más atrajo el interés de Torroja. Sin embargo, no ignoraba éste el avance de las ciencias aplicadas tras la Segunda Guerra Mundial, que hizo que la Astrofísica y la Radioastronomía crecieran en interés e importancia, gracias al poderoso desarrollo de la fotometría y la espectroscopía 7 .

${ }^{6}$ Fernández Pérez, I. (2009). Aproximación histórica al desarrollo de la Astronomía en España. Tesis doctoral leída en la Universidad de Santiago de Compostela.

${ }^{7}$ La fotometría es una técnica que permite a los astrónomos medir el brillo de un astro. Para ello se utilizaba un instrumento denominado fotómetro fotoeléctrico. El primero se construyó en Inglaterra, pero pronto los Estados Unidos tomaron el liderazgo en la construcción de estos instrumentos. En cuanto a la espectroscopía, consiste en una técnica que permite obtener un espectro de la energía (radiación electromagnética) que emiten (o absorben) los objetos celestes y estudiar así la naturaleza química de los mismos. El avance de la espectroscopía fue debido principalmente a una 

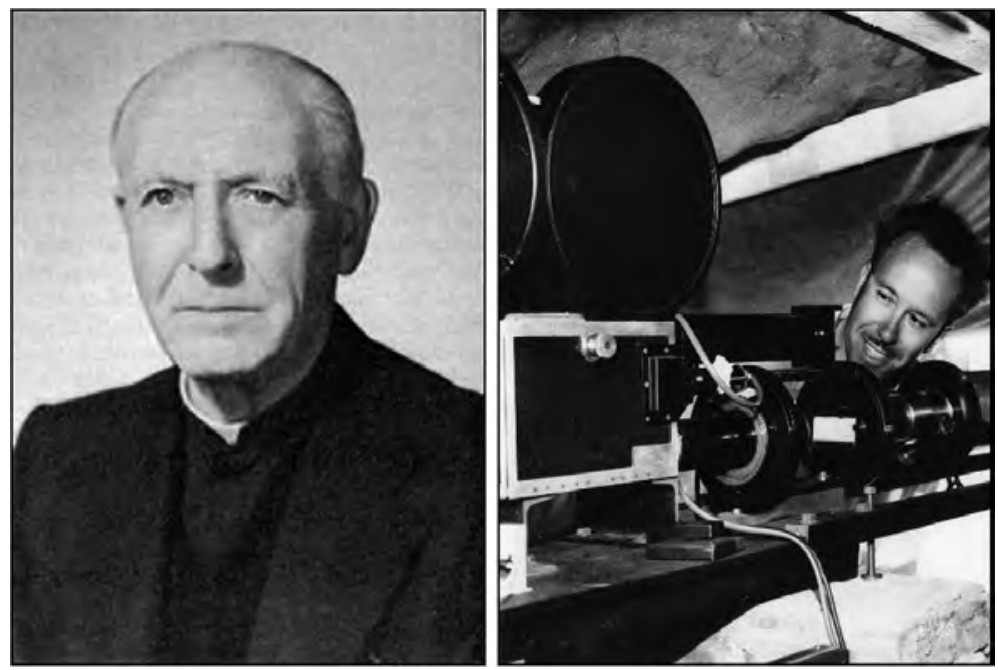

Fotos 1 y 2. Antonio Romañá y José María Torroja.

En cualquier caso, ambos dejaron su impronta tanto en el orden de la investigación como en el docente, así como una escuela de investigadores que serían los protagonistas del nacimiento de la actual astrofísica en España. Prueba de todo ello fue el impulso decidido y creciente que tuvo el OAT desde antes de ver la luz. En unas declaraciones publicadas por el periódico tinerfeño El Día Torroja manifestaba en septiembre de 1959 que este Observatorio del Teide fue una inquietud suya que databa del año 1952. Pasaba entonces por Tenerife en viaje a Guinea con objeto de estudiar un eclipse de Sol -del 25 de febrero-. Cuando visitó la zona de Izańa se le ocurrió que en ella podía montarse un observatorio de extraordinarias posibilidades ${ }^{8}$.

Sin embargo, las condiciones de excelencia para el establecimiento de un observatorio en las cumbres de Tenerife había que demostrarlas no solo en el papel -ya eran conocidas, sin duda, diferentes campańas y citas históricas acerca de su

serie de trabajos de numerosos científicos que culminaron con los resultados obtenidos por Bunsen y Kirchoff sobre los espectros en 1859. Gracias a estos avances, los astrónomos tuvieron una herramienta decisiva para determinar la composición química de los objetos celestes a partir de su espectro, además de otras aplicaciones relevantes.

${ }^{8}$ «He venido laborando en este afán hasta que, hace cerca de un año, ha sido creado por decreto del Ministerio de Educación Nacional. Es éste, en principio, uno de los mejores sitios del mundo para la observación astronómica. El doctor Redman, jefe del Observatorio Astronómico de Cambridge, que estuvo conmigo aquí hace poco más de un año, confirmó mis opiniones. En España existen picos de esta altura, pero zonas ideales tan altas como Izaña, ninguno». El Día, Santa Cruz de Tenerife, 24 de septiembre de 1959. 
emplazamiento desde el siglo XVII- y para ello llegaron a la isla directores e investigadores de diferentes observatorios europeos.

Considérese por otra parte que entre el 1 de julio de 1957 y el 31 de diciembre de 1958 se celebró el Año Geofísico Internacional, en el que España participó tímidamente, exceptuando el Observatorio del Ebro, que se implicó con notable interés. Más de 30000 científicos y técnicos de 66 países cooperaron en una serie de observaciones sobre la Tierra y sus alrededores cósmicos. Solo en 1959, casi 500 cohetes de investigación, 12 satélites científicos y cinco sondas espaciales realizaron importantes observaciones directamente en el espacio, al mismo tiempo que suministraban una nueva perspectiva de la propia Tierra y la baja atmósfera.

Tras dos largas décadas el régimen franquista comenzó a abandonar el modelo autárquico, primero con los «Pactos de Madrid» de 1953 entre España y EE. UU., culminando éstos con la visita del presidente norteamericano Dwight D. Eisenhower a España el 21 de diciembre de 1959. No fue casualidad, por tanto, que una de las catorce estaciones de seguimiento de satélites de la NASA en el planeta fuera instalada en marzo 1960 en Maspalomas (Gran Canaria), otra en 1964 en Robledo de Chavela (Madrid) o la estación de lanzamiento de satélites de El Arenosillo (Huelva) en 1966.

En la memoria del CSIC de los años 1955-1957, se detallaron los que seguramente fueron los precedentes del OAT. Se explica cómo fue oficialmente delegado por la Unión Astronómica Internacional el astrónomo alemán Prof. H. Von Kluver, del Observatorio de Cambridge (Inglaterra), para visitar las islas de Tenerife, Gran Canaria y Fuerteventura y elegir el emplazamiento más adecuado para las misiones extranjeras que tenían en proyecto observar desde dichas islas el eclipse total de Sol del 2 de octubre de 1959. En esta visita le acompañó el doctor Torroja, el cual completó el trabajo visitando asimismo los emplazamientos del Sáhara Español situados bajo la zona de totalidad del eclipse. El mismo Torroja aprovechó el viaje para visitar el Observatorio de Izańa y hacer un primer estudio de sus condiciones para la instalación en el mismo lugar de un "Observatorio Astronómico de Altura", comprobando sus magníficas condiciones de pureza de cielo, latitud y altitud (2380 metros), lo mismo que su facilidad de comunicaciones con los puntos importantes de la isla'.

En la siguiente memoria del CSIC, del año 1958, se manifestaba que, en el mes de abril, por invitación de la Unión Astronómica Internacional, el doctor Torroja se trasladaría a su vez al Observatorio de Cambridge para estudiar la conveniencia de instalar un Observatorio Astronómico en Izaña (Tenerife). Como consecuencia de estas primeras conversaciones, en el mes de julio se trasladaron a Izaña Torroja y el director del Observatorio de Cambridge Prof. Redman, para continuar el estudio sobre el terreno ${ }^{10}$.

\footnotetext{
${ }^{9}$ Memoria del CSIC años 1955-1957, p. 588.

${ }^{10}$ Memoria del CSIC año 1958, pp. 292-293.
} 


\section{LA INCLUSIÓN DEL OBSERVATORIO ASTRONÓMICO DEL TEIDE EN EL OBSERVATORIO METEOROLÓGICO DE IZAÑA}

En octubre de 1957 el meteorólogo y jefe del Centro Meteorológico de Canarias Occidental Enrique Cañadas escribía al meteorólogo Felipe Gracia, jefe a la sazón del centro regional de Canarias, rogándole que hiciera las gestiones necesarias para saber la forma de

conseguir metálico [sic], o poder justificar ciertos gastos ocasionados por las visitas de personalidades científicas a Tenerife, con el propósito de permanecer y conocer Izaña; unos bajo el punto de vista meteorológico y otros, recientemente, bajo el aspecto astronómico y geofísico ${ }^{11}$.

De los astrónomos que últimamente habían estado allí citaba a José María Torroja, catedrático de la Universidad Central, y a Kluber [sic] con su esposa, catedrático de astronomía de la Universidad de Cambrigde, ambos con el propósito de instalar diversos aparatos para la observación del próximo eclipse solar que se produciría en 1959.

Así pues, tal y como anunciara Enrique Cañadas casi un año antes, el 28 de mayo de 1958 informaba al director del Servicio Meteorológico Nacional (SMN) que habían solicitado alojamiento en el Observatorio de Izaña para la segunda quincena de junio, y para permanecer durante dos meses aproximadamente, el director y profesor Redman, del Observatorio Astronómico de Cambrigde, y el director del Landessternwarter, Dr. Phil Hans Kienle, este último en unión de dos ayudantes. Además, añadía que

en la visita que el pasado año realizó a dicho observatorio el astrónomo español Torroja, les manifestó que el emplazamiento del mismo era ideal para la realización de observaciones astronómicas, y que así lo haría saber a sus colegas extranjeros, motivo por el cual deseaba alojarse en Izaña el director del Observatorio Astronómico de Cambrigde. Por tanto, le rogaba que le comunicara su autorización para proceder al alojamiento de estos señores que lo interesaban ${ }^{12}$.

${ }^{11}$ Cada visita significaba un gasto inicial de 600 pesetas en transporte, señalaba Cañadas, más una invitación a un almuerzo como mínimo, ya que creía el meteorólogo que «había que corresponder de esta forma al honor de la visita que les hacían, aparte de que a su vez, a la recíproca, solían invitarles a su hotel a un ágape. Concretando, cada visita tenía unos gastos aproximados de 1000 pesetas». Con motivo del Año Geofísico les habían advertido que vendrían más científicos interesados en el próximo eclipse: «Ante estas perspectivas - preguntaba Cañadas- ¿cómo solucionarían estos problemas económicos? $\mathrm{Ni}$ a un taxista ni a un restaurante se les podía pedir factura sin pagarles en el instante. Mucho le agradecería saber una respuesta, porque hasta entonces yo había pagado de mi bolsillo, pero, claro está, no podía seguir en estas condiciones, indefinidamente». Archivo del Observatorio Atmosférico de Izaña (OAI). Véanse estas vicisitudes en De Ory AJAmil, F. (2007). El Observatorio Meteorológico de Izaña. Historia y vida de una institución cientifica española. Ed. Ministerio de Medio Ambiente, Madrid.

${ }^{12}$ Loc. cit. OAI 


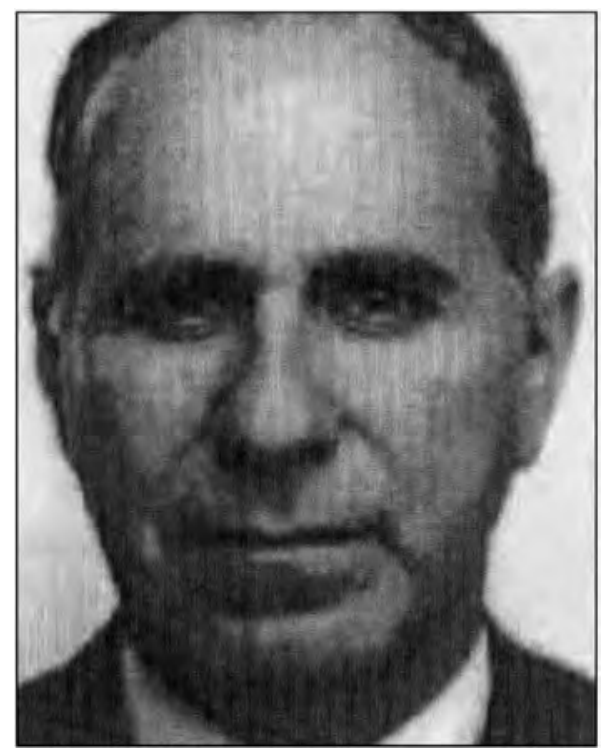

Foto 3. Luis Azcárraga.

En junio de 1958 Luis Azcárraga ${ }^{13}$ se dirigía al general jefe de la Zona Aérea de Canarias, Francisco Mata, para trasladarle la noticia del jefe del Centro Meteorológico de Tenerife. Indicaba que por parte del Servicio Meteorológico Nacional no solo no había ningún inconveniente, sino que la visita de estas personas y el prestigio que con ello podía adquirir el Observatorio le parecía muy conveniente. Le informaba de todo ello para que decidiera si era oportuna la visita de estos señores, y en consecuencia de ello pudiera obtener la correspondiente autorización. Suponía que el jefe de meteorología de la Zona Aérea le habría informado por su parte de la petición. Por su parte ya se lo había dicho también al general subsecretario, y no tenía ningún inconveniente, supuesta su conformidad. Como esta

13 Ingeniero aeronáutico y general de brigada, Luis Azcárraga (Vitoria 1907-Salvatierra 1988) fue asesinado por ETA al salir de una iglesia acompañado de su mujer, recibiendo cuatro tiros por la espalda, dos de ellos en la nuca. Catedrático de la Escuela Superior de Ingenieros Aeronáuticos, fue presidente del INTA, vicepresidente de la OMM, de la ESA. Fue un directivo atípico en la administración de la dictadura, uno de los pocos casos de superación, por sus cualidades personales, del aislamiento internacional en que se mantuvo el régimen durante años, y proporcionó al Servicio Meteorológico Nacional (SMN) una participación en la cooperación meteorológica internacional a través de la OMM que ni antes ni después se volvió a disfrutar. Fue director del SMN desde 1940 a 1969, esto es, veintinueve años. 
era la primera ocasión desde hacía años en que se les presentaba una petición de este orden, y lo natural era que otras sucesivas vinieran otra vez de la dirección del servicio puesto que era el que internacionalmente se conocía, si le parecía bien seguiría el mismo trámite, es decir que cuando recibiera alguna petición, antes de contestarla, le pondría al corriente de ella para que le dijera si había alguna dificultad desde el punto de vista de la «Región» $[\text { sic }]^{14}$.

Ese mismo día, 6 de junio de 1958, Azcárraga se dirigió a Cañadas para prevenirle que

en cuanto al trato con estos señores debía atenerse a lo siguiente: una vez recibiera la autorización del general Mata podía dirigirse a ellos, en respuesta a la carta que de ellos hubiera recibido, expresándoles su conformidad con la llegada y diciéndoles que con alguna anticipación le comunicaran la fecha de la misma ${ }^{15}$.

Convenía también, señalaba el director del SMN, que se lo dijera cuanto antes, y le enviara a Azcárraga las señas de los interesados, o mejor aún copia de la carta que recibiera, con el fin de que éste pudiera escribirles, porque le parecía que era lo más correcto. En lo que se refería a las atenciones para con ellos,

debía procurar que las habitaciones que fueran a utilizar estuvieran bien presentadas y bien atendidas constantemente. Debía comprobarlo Cañadas personalmente, sin fiarse de nadie [sic], y a su vez instruir a las personas del servicio que vivían permanentemente en Izaña para que supieran la forma como debían comportarse $[\mathrm{sic}]^{16}$.

En lo referente a los gastos que hicieran estas personas en Izaña, por supuesto ellos debían pagarlos. Como no tenían ningún régimen establecido para estas visitas, le agradecería a Cañadas que

pensara en este aspecto de la comida y demás gastos, y si tenía alguna duda respecto a la forma de tratarlo con los huéspedes, se lo dijera a Azcárraga con el fin de que pudiera darle su opinión. Siempre sobre la base de que ellos debían pagar sus correspondientes gastos ${ }^{17}$.

El 7 de junio de 1958 el general subsecretario comunicaba al director general de protección de Vuelo la autorización cursada al general jefe de la Zona Aérea de Canarias para dar alojamiento a los científicos extranjeros ${ }^{18}$.

${ }^{14}$ Loc. cit. OAI.

${ }^{15}$ Ibidem.

${ }_{16}$ Ibidem.

17 Ibidem.

${ }_{18}$ Cañadas, a su vez, se dirigía a Azcárraga el 11 de junio de 1958, en respuesta a su carta del 6 de junio, comunicándole que «estaban preparando los enseres necesarios para completar la dotación del observatorio con el fin de que los visitantes se llevaran la mejor impresión. En lo referente al trato con aquellos señores, en general el personal de Izaña estaba ya preparado. Eran res- 


\section{LA CREACIÓN DEL OBSERVATORIO ASTRONÓMICO DEL TEIDE}

Una orden ministerial del Ministerio de Educación Nacional de 10 de febrero de 1959, publicada el 16 de marzo, daba carta de naturaleza a dicho observatorio. En su exposición de motivos se cita que la "zona del macizo del Teide» - no así la de Izaña- parecía reunir magníficas condiciones para la instalación de un observatorio astronómico. Aunque habían sido varios los astrónomos nacionales y extranjeros que en distintas ocasiones expusieron su opinión en este sentido, antes de proceder a su instalación definitiva era conveniente una instalación provisional que permitiera, al mismo tiempo que un detenido estudio de las condiciones de aquella zona, la elección del lugar preciso para su emplazamiento definitivo.

Dada la extensión de la citada orden ministerial se referirán solo los artículos citados en relación con el OMI. Así, el artículo sexto establecía que dicho ministerio atendería a los gastos de instalación y sostenimiento del observatorio con cargo a los créditos presupuestarios que pudieran ser dedicados a dicha finalidad. El artículo siguiente cifraba un plazo de dos ańos, a partir de la fecha en que se iniciara el funcionamiento del observatorio, para que el patronato elevase a ese departamento un informe sobre la organización definitiva que debiera darse al mismo a la vista de los resultados de las observaciones y estudios previos realizados durante el indicado periodo. Y finalmente, pero no menos importante, el artículo octavo ordenaba que «provisionalmente, el Observatorio del Teide se instalará, con la debida autorización del Ministerio del Aire, en los terrenos del Observatorio Meteorológico de Izaña ${ }^{19}$.

Otra orden ministerial de 18 de junio de 1959, BOE de 3 de agosto, nombraba director del Observatorio del Teide, por propuesta del rector y presidente del patronato, a José María Torroja Menéndez, catedrático de Astronomía General y Topografía y Astronomía Esférica y Geodesia de la Universidad de Madrid.

Para apreciar la imperante atmósfera militarista de aquellos años, otra orden de 26 de octubre de 1959, BOE de 9 de diciembre, ampliaba el artículo segundo de la primera de febrero, incluyendo al general jefe de la Zona Aérea de Canarias como máxima autoridad del Ejército del Aire en el archipiélago en el patronato que regía el OAT -formado por autoridades locales y académicas de la Universidad de La Laguna-.

petuosos y atentos ya que estaban acostumbrados, por el mucho tiempo que llevaban, a tratar con diversas personalidades [sic]. Las noticias que habían tenido de estos señores habían sido por cartas particulares que les había dirigido el astrónomo Dr. Torroja, catedrático de la universidad central de Madrid, quien podía informarle sobre los propósitos e investigaciones que deseaban llevar a cabo los visitantes. Y efectivamente, ya habían pensado que los gastos que hicieran estas personas en Izaña serían por cuenta de los interesados. No debía preocuparse Azcárraga por el adecentamiento de las habitaciones ni por el servicio, pues ya vería cómo los referidos astrónomos se llevarían la más grata impresión». Ibidem.

19 BOE de 16-02-1959. 
Por otra parte, el OAT se concibió como un centro científico interuniversitario, dedicado fundamentalmente a la investigación astronómica, abierto a todas las universidades españolas, que podrían enviar personal propio y mantener programas propios de investigación en el Observatorio. El OAT se comprometía a colaborar en la labor docente de las facultades de ciencias de las universidades españolas, ofreciendo a su propio personal científico para dar cursos monográficos sobre los temas de su especialidad ${ }^{20}$. Sin embargo, la escasez de personal y medios era flagrante desde sus inicios. La Sección de la Alta Atmósfera y el Medio Interplanetario, que comenzó a funcionar en 1964, reunía en torno a ella en 1970 a tres astrónomos y dos ayudantes de observación ${ }^{21}$.

Al final del artículo citado el propio Francisco Sánchez - quien posteriormente llegara a ser director del IAC- manifestaba que «nuestro problema fundamental es la falta de personal científico preparado, capaz de extraer resultados del gran almacén de datos-observaciones que vamos consiguiendo $»^{22}$.

\section{LAS PRIMERAS ACTIVIDADES DEL OBSERVATORIO ASTRONÓMICO DEL TEIDE EN EL OBSERVATORIO METEOROLÓGICO DE IZAÑA}

Quedó suficientemente aclarado que los desembolsos ocasionados por las visitas de investigadores extranjeros no serían costeados más por el SMN. Sin embargo, numerosos gastos referidos a las infraestructuras del nuevo OAT sí fueron sufragados por el SMN. Así lo reflejaba Torroja en la carta dirigida a Cañadas en abril de 1959, donde le comunicaba la autorización de Azcárraga para la construcción de un pabellón para la instalación de un telescopio ecuatorial en Izaña, así como la conducción eléctrica desde el OMI hasta el mismo.

Además, las visitas de investigadores extranjeros al OMI tampoco cesaron en lo sucesivo. Desde los meses de abril a julio de 1959 bajo la dirección de Hans Kienle, director del Observatorio de Heidelberg, se establecieron en el OMI el doctor Dietrich Labs, auxiliado por H. Neckel y R. Rinklef, con el objetivo de determinar la idoneidad del cielo para las observaciones mediante el estudio de la distribución de la intensidad espectral absoluta del Sol.

20 Torroja, J.M. ${ }^{a}$ y Sánchez, F. (1970). El nuevo Observatorio del Teide, su organización y puesta en marcha. Urania, números 271-272, p. 94. Barcelona.

${ }_{21}$ Ibidem. p. 119.

${ }^{22}$ La segunda Sección de Física Solar estaba a cargo del jesuita Juan Casanovas -quien acabó su carrera como director de la Specola Vaticana, u Observatorio del Vaticano- y no comenzó a funcionar hasta agosto de 1969, cuando se instaló un heliógrafo Razdow SW-250, para lo que hubo que construir una torre de 13 metros. El cable subterráneo que suministraba corriente eléctrica a ambas secciones procedía de la estación emisora de Televisión Española, distante un poco más de un kilómetro. A finales de 1970 el personal que llevaba a cabo las observaciones eran el propio astrónomo y un observador como ayudante. Cfr. Casanovas (1970). El nuevo Observatorio del Teide. La Sección de física solar. Urania, números 271-272, p. 126. Barcelona. 


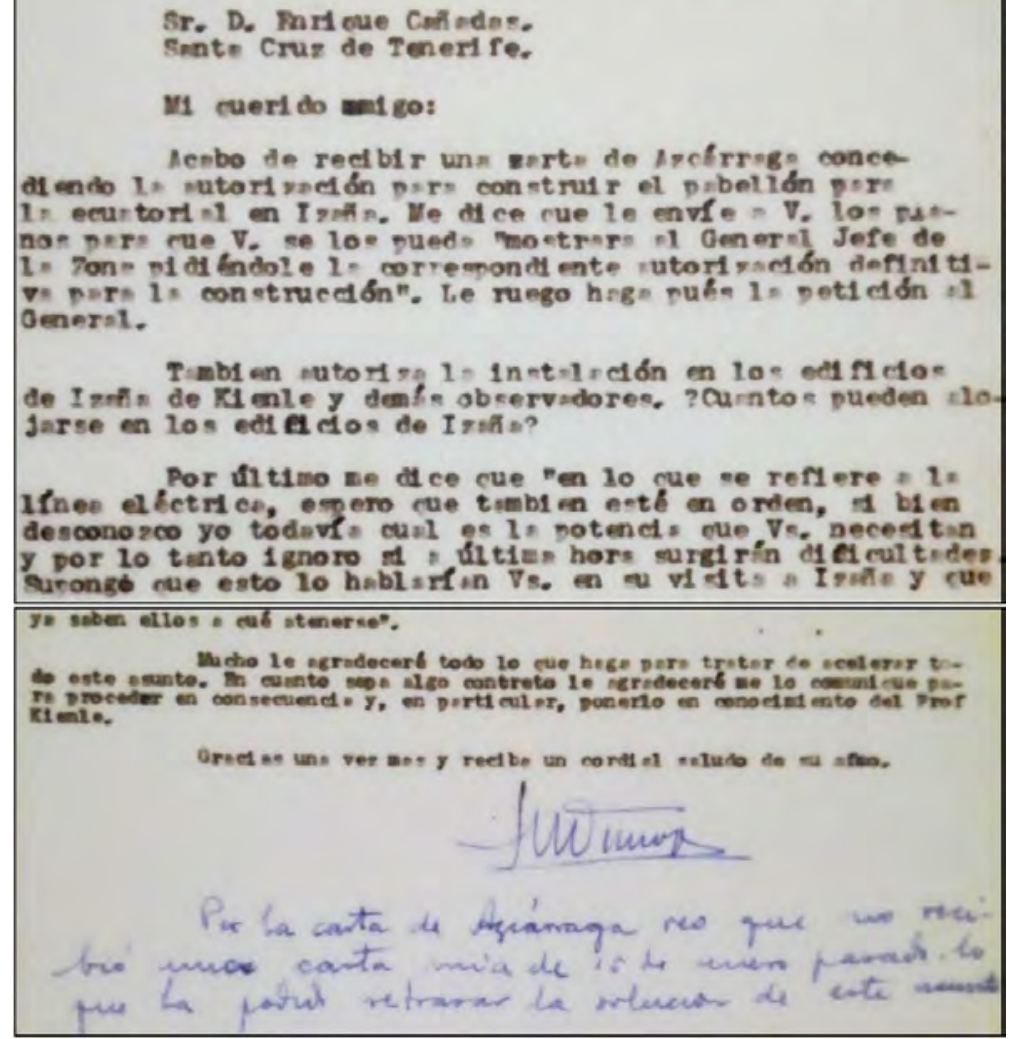

Foto 4. Carta de José María Torroja a Enrique Cañadas. Archivo OAI.

Pero una intensa y prolongada intrusión de polvo sahariano fue determinante para emitir un informe negativo, dirigiéndose el proyectado observatorio alemán a las cumbres de Calar Alto, en Almería (hoy Instituto de Astrofísica de Andalucía).

Del mismo modo, en una carta de agosto de 1959 un ingeniero del Instituto Geográfico Nacional solicitaba autorización para alojar en el OMI a un investigador norteamericano interesado en realizar ionosondeos de la alta atmósfera, con motivo del eclipse del 2 de octubre de 1959.

Pocos días después, en septiembre de 1959, José María Torroja visitaba Izaña en ruta hacia el África occidental española, para inspeccionar el montaje de los aparatos del OAT y la puesta a punto de su instalación para el próximo eclipse de octubre. De acuerdo con la orden de 10 de febrero, antes de construir instalaciones definitivas era preciso hacer un estudio detallado para comprobar si las condiciones del lugar eran idóneas para el emplazamiento de un observatorio astronómico. Para ello 


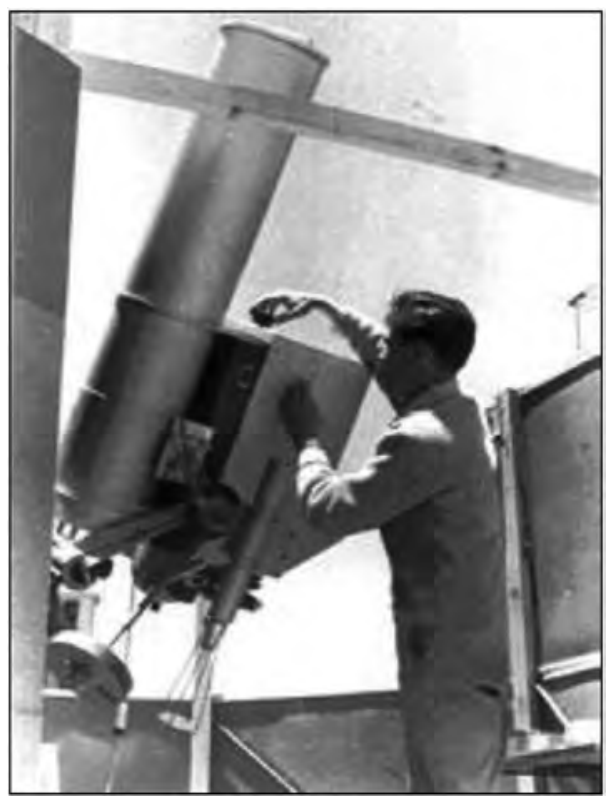

Foto 5. D. Labs y el espectrofotómetro solar.

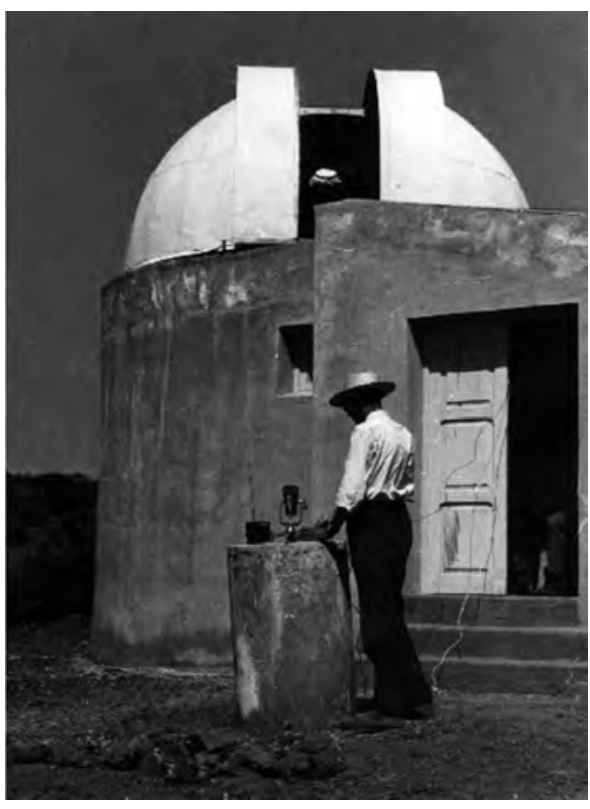

Foto 6. F. Molina en el pabellón ecuatorial. Primer observatorio del IAC.

era necesario trasladar a Izaña a un investigador español que realizara mediciones de la calidad del cielo con el uso de técnicas más modernas.

Torroja elige para dicha tarea a un joven físico, Francisco Sánchez Martínez. Sánchez nació en Toledo el 16 de mayo de 1936 y estudió ciencias físicas en Madrid. A finales de los años cincuenta, marcado por el perfil de pluriempleado de la época, Sánchez investigaba en el Instituto de Óptica Daza de Valdés del CSIC y era profesor de prácticas en la Cátedra de Óptica de la Universidad Complutense, además de profesor suplente en el Instituto Ramiro de Maeztu. Pues bien, en enero de 1961, se trasladó a Tenerife contratado por la Universidad de La Laguna. Durante sus primeros años su labor consistió en recopilar información y adquirir experiencia en investigación sobre prospección del cielo para medir su calidad. Afirmaba Sánchez que ya había nacido el European Southern Observatory (ESO) en Chile, donde se estaban haciendo las prospecciones y que de ellos aprendió. También estuvo formándose gracias a una ayuda de la IAU en el Instituto de Astrofísica de París y en el Observatorio de Alta Provenza ${ }^{23}$. 

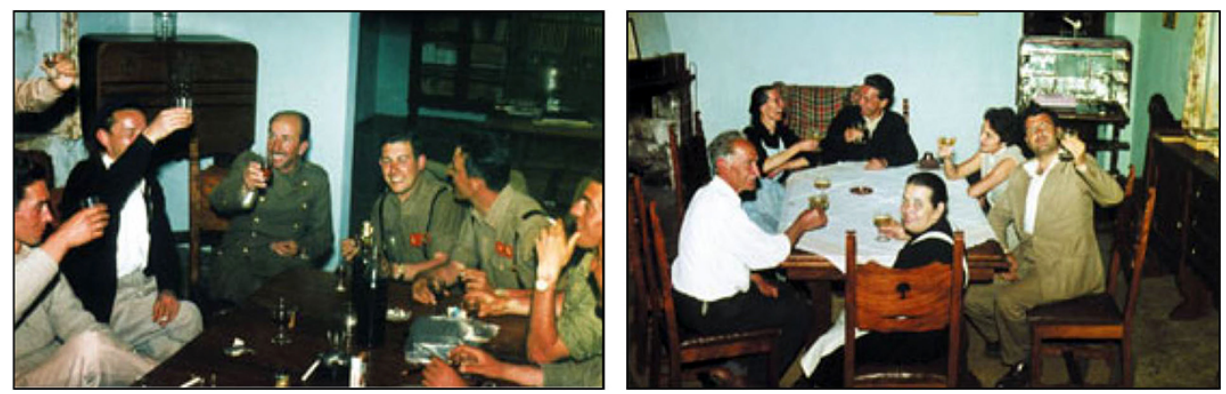

Fotos 7 y 8. Despedida de Rinklef y Labs de un grupo de militares y del personal del OMI en julio de 1959.

Francisco Sánchez residió con su familia en el OMI durante más de cinco años $^{24}$ y en 1961 comenzó la prospección previa al montaje del OAT. Basándose en los datos del OAI registrados desde 1944 a 1966, concluyó que

las condiciones atmosféricas locales eran muy favorables para un emplazamiento astrofísico: [hay] gran número de días al año en que se puede observar, transparencia generalmente cercana a la atmósfera teórica pura y seca, y también la calidad de las imágenes astronómicas parece buena ${ }^{25}$.

Para refutar las opiniones desfavorables de algunos astrónomos extranjeros por las irrupciones de polvo sahariano en Izaña, Sánchez utilizó los datos climatológicos del OAI y se valió del trabajo del meteorólogo Inocencio Font Tullot El tiempo atmosférico en las islas Canarias ${ }^{26}$.

La precariedad de medios le llevó a manifestar

Tuve que determinar qué había que medir, y diseñar los instrumentos correspondientes, algunos de los cuales construí personalmente. Era una labor polifacética, muy instructiva. Tenía que observar de día y de noche; y era necesario, además, estudiar y estar dispuesto a cualquier actividad extra por imprevista que fuera. Como cavar una zanja, sacar agua del pozo, partir leña... ${ }^{27}$.

Una muestra de su quehacer diario -o nocturno por mejor decir- lo manifestaba en una carta dirigida al jefe del OMI en noviembre de 1963. Pero Sánchez no

${ }^{24}$ Lozano Martín (2009). Historia del Departamento de Astrofisica de la Universidad de La Laguna. Edita: Instituto de Astrofísica de Canarias y Departamento de Astrofísica de la Universidad de La Laguna.

\footnotetext{
25 Sánchez Martínez. Loc. cit. p. 196.

26 Ibidem. p. 199.

27 Lozano Martín. Loc. cit.
} 


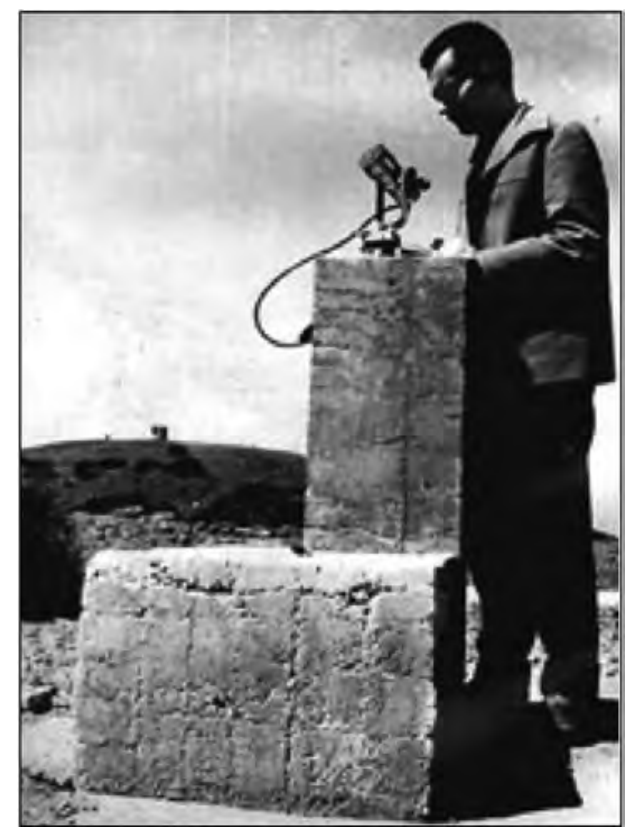

Foto 9. Francisco Sánchez con un pirheliómetro en Izaña. c. 196.

estuvo realmente tan solo para el desempeńo de sus labores en el OAT. Pedro Rodríguez García-Prieto, jefe del OMI al que Francisco Sánchez dirigía su carta, relataba a su vez y muy expresivamente el estado en que se encontraba el OAT en $1962^{28}$. De este modo Sánchez, tras unos meses de preparación, comenzó en el OMI sus estudios de prospección de calidad del cielo en enero de 1962. Ese mismo

${ }^{28}$ En una carta privada dirigida al meteorólogo José Frías Pasuti, entonces jefe del personal del SMN, aquél le comentaba a éste que «los de astronomía seguían con su situación provisional desde hacía tres años, y no veía ningún movimiento que le indicara que iban a tomar la independencia. Cada día aumentaba su número y con ello el trabajo que daban al personal del observatorio. A las mujeres las gratificaban a instancia suya, y daban una pequeńa limosna a los informadores que colaboraban con ellos. Hacía poco - prosigue Rodríguez- se habló de un plan de colaboración oficial intensa, por el que todos pasarían a colaborar en astronomía y este observatorio quedaría afecto al meteorológico. Pero no había nada por el momento. Pensaba un día hacer un informe sobre la cuestión y enviarlo al director del astronómico, y a él como jefe de personal para resolver de una vez todos los puntos que estaban surgiendo cada día. La verdad es que ellos procuraban dar la menor guerra posible y las relaciones eran buenas, como correspondía entre gente civilizada. Pero lo que no le gustaba es que todo el mundo se desentendiera de ellos, y fuera él quien tuviera que resolver todas las pegas a costa a veces de resoluciones personales que algún día le criticarían o intentarían desautorizarle. Había que tener en cuenta que eran unos invitados que llegaron hace tres ańos con las manos en los bolsillos, y seguían de invitados [sic]». Loc. cit. OAI. 


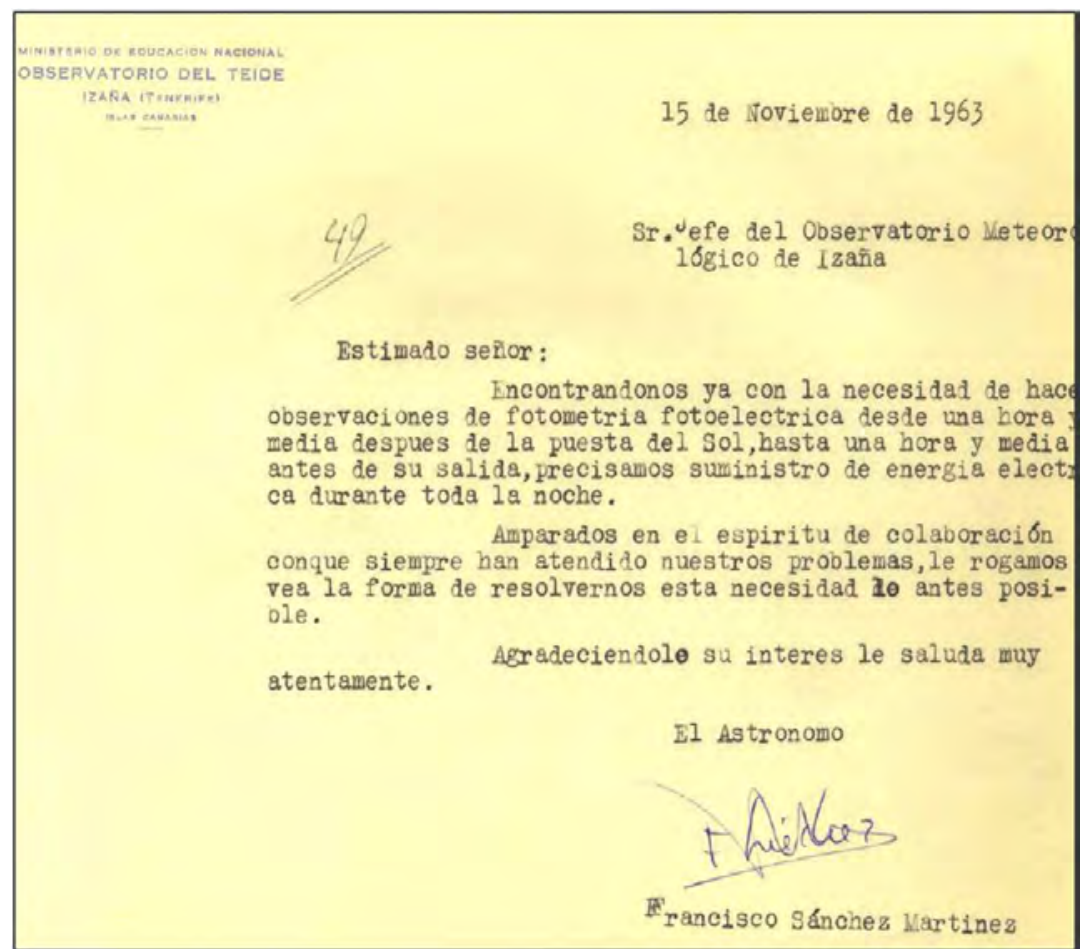

Foto 10. Carta de Francisco Sánchez a Pedro Rodríguez García-Prieto. Archivo del OAI.

mes Jean Dommanget, astrónomo del Observatorio de Uccle (Bélgica), especialista en este tipo de trabajos, pasó cuatro semanas en Izaña, y en agosto de 1962 René Dumont, del Observatorio de Burdeos, visitó el OMI para conocer in situ la posibilidad de realizar observaciones de la luz zodiacal. Posteriormente realizó una estancia de varios meses, hasta que en enero de 1964 llegó al OMI su fotopolarímetro espectral para el estudio de la luz zodiacal. También en el año 1967 ocurrió la llegada del jesuita Juan Casanovas, del Observatorio del Ebro, con el fin de investigar las posibilidades de la zona para llevar a cabo observaciones solares.

\section{LA AUTONOMÍA DEL OAT Y LOS PLANES ECONÓMICOS DE DESARROLLO}

Para ir finalizando deben citarse algunos matices acerca de la situación político-económica de la administración franquista del Estado y los medios del OAT para su emancipación del OMI. Se ha dicho que la independencia económica y comercial 


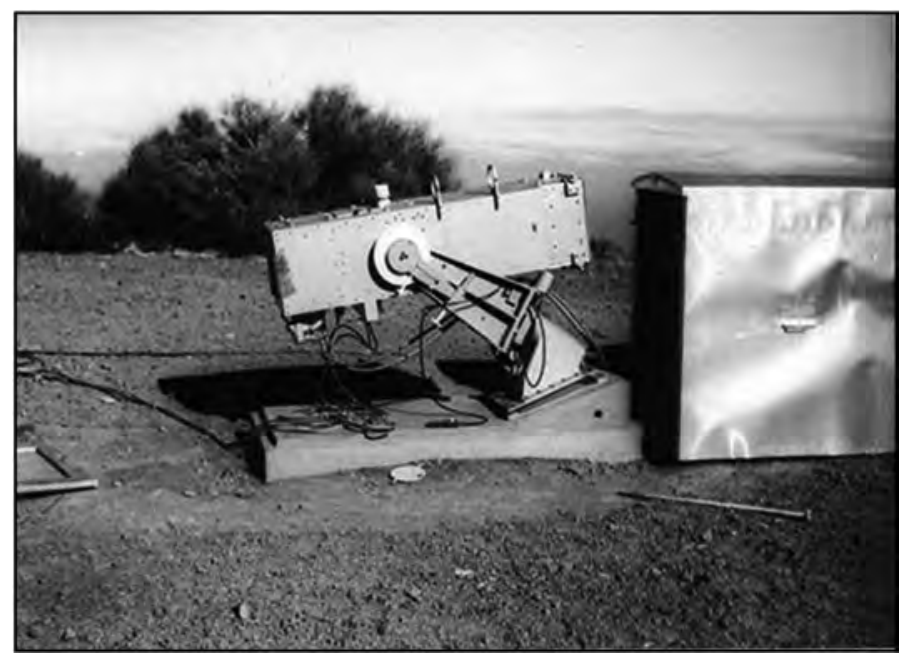

Foto 11. Fotopolarímetro de René Dumont.

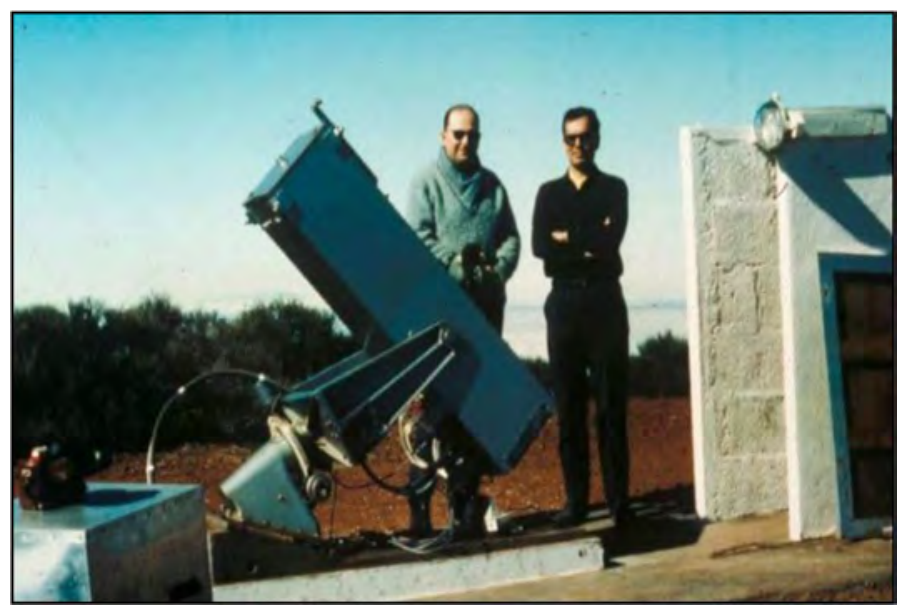

Foto 12. René Dumont y Francisco Sánchez.

del exterior y la férrea intervención del Estado caracterizaron el periodo de autarquía económica que abarcó desde 1936 hasta 1951 o 1957, según diferentes autores.

Baste decir que las cifras macroeconómicas y el nivel de vida del país sufrieron una fortísima recesión, situándose por debajo de los resultados del primer bienio de la II República. El cambio del gobierno de Franco en 1957 facilitó un giro en la dirección de la política económica. El Plan de Estabilización Económica fue 


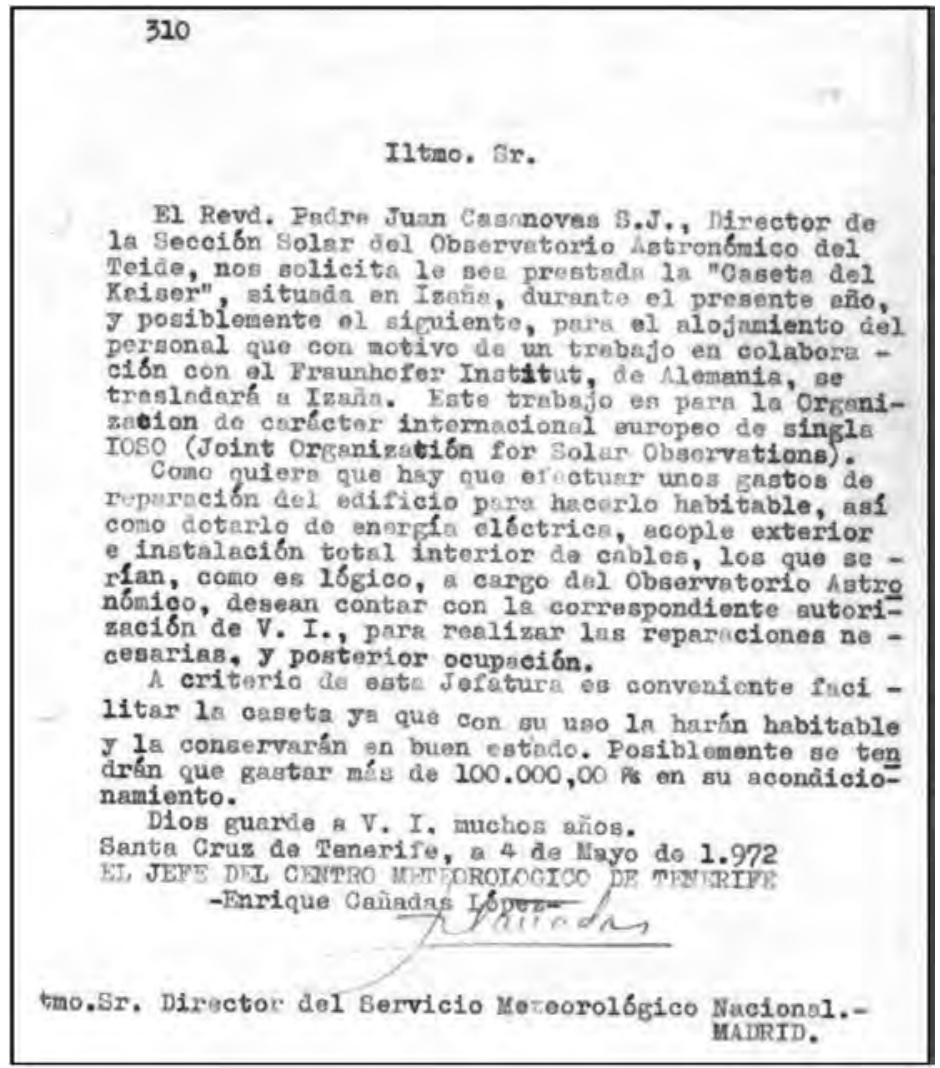

Foto 13. Carta de Juan Casanovas a Luis Azcárraga.

el resultado de las nuevas ideas que aportaron al régimen franquista los «tecnócratas", un grupo de jóvenes economistas cercanos al Opus Dei.

Pues bien, en la creación de la comisaría de los Planes de Desarrollo (de 1964-1967 y 1968-1971) fue donde el OAT encontró la financiación necesaria para su autonomía y desarrollo. Así la «Propuesta de Organización del Observatorio Astronómico del Teide», según el proyecto de Torroja, fue presentada en 1966 a la Comisión de Investigación del II Plan de Desarrollo ${ }^{29}$, encargada de crear polos de industrialización en diferentes lugares del país. El coste total del proyecto (edificios, equipos, personal y sostenimiento) llegaba a más de 230 millones de pesetas a repartir en cuatro anualidades.

29 Véase con más detalle en Fernández Pérez. Loc. cit. p. 159. 
De tal modo fue como el OAT alcanzó su independencia y autonomía económica. Pero aun así y con todo, en los años siguientes el OMI siguió prestando su colaboración y ayuda al OAT, como lo demuestra la carta del año 1972 en la que a petición del astrónomo Casanovas, Cańadas solicitaba permiso para ceder la caseta del Kaiser a una comisión del Fraunhofer Institut de Friburgo (RFA).

\section{CONCLUSIONES}

La que es hoy una de las tres mejores instituciones mundiales en materia de astrofísica -junto con Hawai en EE. UU. y Atacama en Chile- tuvo unos comienzos muy arduos y precarios, como cabría esperar de los años en los que se dio su creación y se ha documentado oportunamente. Ello demuestra que no puede desvincularse la historia de la ciencia del contexto más general de la historia y la sociedad. Cabe hoy preguntarse si hubiera sido posible antes o después de entonces su creación. La respuesta es sencilla si se considera la coyuntura político-económica y académica de la España de los años 50 y 60. Lo más probable es que se hubiera dado bastantes ańos después de aquel eclipse de 1959, pero seguramente nunca antes. Porque sin la colaboración material y del personal del OAI, y sin el aprovechamiento de su base de datos climatológicos, es más que probable que su validación como lugar de excelencia para la observación astrofísica se hubiera demorado unos cuantos años más.

Más allá de las instituciones, es obligado destacar el buen oficio y el interés mostrado por las personas que lo hicieron posible. Sin el concurso de personalidades como las de Torroja y Azcárraga, Cañadas y Sánchez, no es posible imaginar la creación de un observatorio astrofísico al que, además de las adversidades citadas, hay que añadir que fue el primero de su disciplina en España. En ello coincidió paradójicamente con su vecino, el Observatorio Meteorológico de Izaña.

El Instituto de Astrofísica de Canarias (IAC) fue creado definitivamente el 16 de septiembre de 1975, mediante un acuerdo del CSIC con la Universidad de La Laguna y los cabildos insulares. Pero fue en 1973 cuando el OAT pasó a depender del Instituto Universitario de Astrofísica creado por la ULL, cobrando un verdadero impulso con la creación de la especialidad universitaria de Astrofísica, la primera de España. Siendo Francisco Sánchez el primer catedrático de la disciplina en Espańa, con la experiencia acumulada y los investigadores extranjeros ya establecidos en sus propios observatorios, el IAC comenzó su singladura en el plano internacional alcanzando metas y éxitos insospechados entonces y aún hoy. 
